\title{
Nicotine lozenges and behavioural interventions may help smokeless tobacco users to quit
}

\author{
Abstracted from
}

Ebbert JO, Elrashidi MY, Stead LF.

Interventions for smokeless tobacco use cessation. Cochrane Database Syst Rev 2015; 10: Art. No. CD004306. DOI: 10.1002/14651858.CD004306.pub5.

Address for correspondence: Jon O Ebbert, Division of Primary Care Internal Medicine, Mayo Clinic, 200 1st Street Southwest, Rochester, Minnesota, 55905, USA. E-mail: ebbert.jon@mayo.edu.

\section{Question: How effective are behavioural and pharmacotherapeutic interventions in treating smokeless tobacco use?}

Data sources Cochrane Central Register of Controlled trials (CENTRAL); Medline; Embase; PsycINFO. Additional sources were also searched for early versions of the review: Web of Science, Dissertation Abstracts Online, Scopus, Healthstar, ERIC, National Technical Information Service database and Current Contents.

Study selection Randomised controlled trials and pseudo-randomised controlled trials allocating smokeless tobacco (ST) users to an intervention or control, or to different interventions. Interventions could be pharmacological (ie nicotine replacement therapy (NRT), bupropion, varenicline) or behavioural, and could be directed at individual ST users or at groups of users.

Data extraction and synthesis Two authors independently extracted data about participants, interventions, outcomes and methodological quality. Any discrepancies in extracted data were resolved by consensus. Synthesis followed standard Cochrane methodology. Results Thirty-four trials were included, 32 from the USA, one from Sweden and one from Sweden and Norway. Sixteen of the trials assessed pharmacological interventions and 19 assessed behavioural interventions (two studies did both). Varenicline increased ST abstinence rates (risk ratio 1.34, 95\% confidence interval (Cl) 1.081.68). Bupropion did not show a benefit of treatment. Neither nicotine patch nor nicotine gum increased abstinence. Nicotine lozenges did increase tobacco abstinence (RR 1.36, 95\% Cl 1.17-1.59). Behvaioural interventions resulted in a RR of $1.39(95 \% \mathrm{Cl} 1.25-1.55)$ for those already motivated to quit and $1.37(95 \% \mathrm{Cl} 1.23-1.53)$ for anyone. Conclusions Varenicline, nicotine lozenges and behavioural interventions may help ST users to quit. Confidence in results for nicotine lozenges is limited. Confidence in the size of effect from behavioural interventions is limited because the components of behavioural interventions that contribute to their impact are not clear.

This paper is based on a Cochrane Review published in the Cochrane Library 2015, issue 10 (see www.thecochranelibrary.com for information). Cochrane Reviews are regularly updated as new evidence emerges and in response to feedback, and the Cochrane Library should be consulted for the most recent version of the review.

\section{Commentary}

Smokeless tobacco is used by more than 300 million people, $90 \%$ of whom live in Southeast Asia. ${ }^{1}$ Whilst I would imagine most in the dental profession are familiar with the health consequences of smoking tobacco, some may be less familiar with those encountered by users of smokeless tobacco.

The evidence around smokeless tobacco and oral and general health is less substantial than it is for smoked tobacco and where there is evidence it seems to be contradictory. A recent systematic review and meta-analysis suggested that use of smokeless tobacco in Scandinavia had no effect on oral cancer rates, for example, and yet in the USA the relative risk was 2.6 (that is, smokeless tobacco users were 2.6 times more likely to get oral cancer than non-users). ${ }^{2}$ In another review of longitudinal observational studies in Southeast Asia the odds ratio of getting oral cancer from chewing smokeless tobacco was 2.9 when only cohort studies were included, ${ }^{3}$ which is not too dissimilar to the US relative risk (when the odds ratio is low it approximates the relative risk).

Relatively few studies appear to have looked at the impact of smokeless tobacco use on other health conditions, ${ }^{4}$ though one prospective cohort study found that smokeless tobacco use in a Swedish population was associated with a relative risk of 1.4 (95\% CI 1.2-1.6) for cardiovascular disease compared to non-users. ${ }^{5}$

This systematic review is well conducted and helps us to understand the available interventions that have been tested using randomised controlled trials and pseudo-randomised controlled trials, and that followed up participants for over six months. Nine of the 34 studies included have been reported since the last update in 2011 and there is one trial that is ongoing. There seems to be a lot of activity in this field but, as we will see later, there appears to be a lack of research in the countries that have the most to gain from effective interventions.

\section{Pharmacological interventions}

The majority of these studies included a behavioural intervention in both the intervention and control arms. Thus the controls were not 'no treatment', but included in themselves what might well have been an effective intervention (see behavioural interventions below).

Bupropion, an antidepressant, has been shown to be effective in helping smokers to quit ${ }^{6}$ but in this review the two studies found no effect on smokeless tobacco use quitting.

Nicotine lozenges did show an improvement in quit rates with a relative risk of 1.36 (95\% CI 1.17-1.59). That is, those using lozenges compared to placebo were $36 \%$ more likely to give up. 
Nicotine gum and patches did not tend towards a significant benefit over placebo.

Varenecline, which has also been shown to be beneficial in helping smokers quit was similar in effect to the nicotine lozenges, with a relative risk of abstinence of 1.34 (95\% CI 1.08-1.68).

\section{Behavioural interventions}

The seven studies that selected participants who were already motivated to stop using smokeless tobacco had an overall relative risk of abstinence from tobacco of 1.39 (95\% CI 1.25-1.55). In those studies that selected anyone to participate the relative risk was only marginally lower at 1.37 (95\% CI 1.23-1.53).

By including telephone support in the intervention the relative risk increased to 1.77 (95\% CI 1.57-2.00). When an oral examination was combined with telephone support the relative risk increased to 2.07 (95\% CI 1.61-2.66). That is, the likelihood of long-term abstinence is doubled.

These long-term quit rates may seem modest. If we were to assume that the unaided quit rate is two in 100 then by using behavioural techniques an additional one or two smokeless tobacco users in a 100 will give up. In the unrealistic event that these interventions were offered to the 300 million smokeless tobacco users in the world, instead of 1.5 million giving up alone an additional 750,000 to 1.5 million people would benefit.

There is a problem, though. Whilst no fault of the authors of this review, given that more than $90 \%$ of the population using smokeless tobacco lives outside of the USA and Europe, 32 of the studies were conducted in the USA, one in Sweden and one in Sweden and Norway, with the majority of participants male (where gender is reported). This leaves us in a bit of a bind about how to generalise the results of these studies to the many millions of people who use smokeless tobacco and are not a) male or b) American or Scandinavian. The role of culture, the local healthcare system and economics may mean that these interventions are impossible to deliver or would not be acceptable or effective in other groups.
If as a global profession we are to impact the oral and general health of people everywhere then we do need to develop trials that test culturally-nuanced behavioural or pharmacological interventions that are financially and manpower viable in the countries that would benefit most.

In the UK there has been substantial funding for specialist smoking services and in some areas where there are communities using smokeless tobacco there are specialist services to help address these. The UK's National Institute for Health and Care Excellence has produced guidance on how to help Southeast Asian users of smokeless tobacco to quit (https://www.nice.org.uk/guidance/ph39) and Public Health England offers guidance for those involved in commissioning and delivering oral health care to help all tobacco users to quit. (https:/www.gov.uk/government/uploads/system/ uploads/attachment_data/file/288835/SmokeFree_Smiling_110314_ FINALjw.pdf )

Dominic Hurst

Dental Public Health Unit, Barts and The London School of Medicine and Dentistry, Queen Mary University of London, London, UK and Department of Primary Care Health Sciences, University of Oxford, Oxford, UK

1. National Cancer Institute and Centers for Disease Control and Prevention. Smokeless Tobacco and Public Health: A Global Perspective2014 Contract No. NIH Publication No. 14-7983 2014.

2. Boffetta P, Hecht S, Gray N, Gupta P, Straif K. Smokeless tobacco and cancer. Lancet Oncol 2008; 9: 667-675.

3. Khan Z, Tonnies J, Muller S. Smokeless tobacco and oral cancer in South Asia: a systematic review with meta-analysis. J Cancer Epidemiol 2014; 2014: 394696. doi:10.1155/2014/394696.

4. Critchley JA, Unal B. Health effects associated with smokeless tobacco: a systematic review. Thorax 2003; 58: 435-443.

5. Critchley JA, Unal B. Is smokeless tobacco a risk factor for coronary heart disease? A systematic review of epidemiological studies. Eur / Cardiovasc Prev Rehabil 2004; 11: 101-112.

6. Hughes JR, Stead LF, Hartmann-Boyce J, Cahill K, Lancaster T. Antidepressants for smoking cessation. Cochrane Database Syst Rev 2014; 1: CD000031. doi:10.1002/14651858.CD000031.pub4.

Evidence-Based Dentistry (2015) 16, 104-105. doi:10.1038/sj.ebd.6401129 